\title{
A Preliminary Assessment of Physical \& Virtual Presence in Exergames
}

\author{
Luís Duarte \\ Paulo Ribeiro \\ André Rodrigues \\ Universidade de Lisboa \\ Edifício C6,Campo-Grande, 1749-016 Lisboa, Portugal \\ \{lduarte,pribeiro\}@lasige.di.fc.ul.pt \\ andrefprodrigues91@gmail.com \\ Tiago Guerreiro \\ Luís Carriço \\ \{tjvg,Imc\}@di.fc.ul.pt
}

\begin{abstract}
Exercising is an activity in which the presence of others can motivate an individual to surpass his / her own limits. In recent years, technology has changed the way we carry out these activities. User commitment has been shown to be dependent on, among other, peer pressure. The introduction of challengeable virtual opponents broadened the spectrum of possibilities, enabling users to be motivated by either a real or virtual partner. In this paper we present an early assessment of the impact that different types of presence exerts on the users' perceived motivation and competitiveness. In particular, we delve into how virtual entities compare to their real counterparts. We carried out an experiment in which we sought at obtaining amateur athletes perceptions on their motivation and competitiveness when exercising against real and virtual opponents. Results serve not only the purpose of showing that different types of presence counterbalance each other but they also validate a design space for physical and social partners in exertion applications in which our hypothesis are based upon.
\end{abstract}

Exergames. Persuasion. Presence.

\section{INTRODUCTION}

Sedentary lifestyles are known to originate health conditions that could be prevented or even minimized by exercising a few hours a day [Mueller 2009]. The increasingly pervasive nature of work in our lives often inhibits individuals from spending a few minutes a day exercising, attempting to improve their physical and cognitive conditions. Unfortunately, many do not have the time to spend exercising, either because they are working late or because there are other matters that take that little time away. Driven by this increasingly worrying issue, researchers and developers began capitalizing on modern smart-phones. Their feature sets (e.g. GPS tracking, health sensor add-ons or high quality cameras) and ubiquitous nature allowed for the creation of applications which aid users in tracking their exertion activities. These tools are typically referred to as exergames, since they capitalize on videogame features and characteristics to entice users. Examples of such applications are Endomondo Sports Tracker ${ }^{1}$, Nike ${ }^{2}$, Adidas miCoach ${ }^{3}$ or Runtastic ${ }^{4}$. Exergames are typically used to support exercise activities,

\footnotetext{
${ }^{1}$ http://www.endomondo.com

2 http://nikeplus.nike.com/plus/products/gps_app/

${ }^{3}$ http://www.adidas.com/us/micoach/

${ }^{4}$ http://www.runtastic.com/
}

mostly related with running and jogging. They also sport a variety of features for users to analyse their performance during or after workout sessions as well as comparing their data with their friends'. Ultimately, these features can be categorized as persuasive elements as they effectively motivate the user for the current or future exertion activities.

The advent of social networking features has given relevance to a particular set of these features: the ability to compare oneself to another (Chittaro 2012). Exergames often integrate functionalities which allow users to compare themselves to others whether offline or during the race by loading specific workout data. If being compared online, the user is steadily fed with information regarding whether his / her performance outmatches the recordings'. Additionally, some exergames now offer the option to track and monitor live workout sessions, enabling audience members to intervene by cheering for their friends. Despite representing a popular feature set for this type of applications, knowledge regarding how individuals working out feel when prompted with feedback from observers or when being compared to another user's recording is scarce. Furthermore, the way this information is conveyed can also be a pivotal factor into more successfully reaching a user.

Following the definition of a design space for the creation of exergames containing notions of 
physical and virtual presence (Duarte 2014) we sought to assess how users are influenced by these features in this type of applications. This paper reports our initial experimentation on this topic. Despite the limitations of our experimental settings, our ongoing research is reinforced by positive hints retrieved within the context of these trials. We aimed at a subjective assessment in which the users' perceived motivation and competitiveness level were the main targets. We addressed how the presence of a real opponent, a virtual opponent and a virtual audience impact on an individual.

\section{RELATED LITERATURE}

The diversity of existing exergames testifies to the importance and popularity of this type of software. These applications sport a significant feature set, allowing the users to configure options such as the course they desire to tackle or defining personal challenges. Among these characteristics, the usage of persuasive mechanisms is a common trait - if the application is not able to motivate users into exertion activities, then it failed its purpose. We will first provide a brief overview of exergames and then delve into a specific approach utilized to motivate users based on social dynamics.

\subsection{Bridging Exercise \& Entertainment}

Exergames provide an excellent set of advantages that range from contributing to weight loss and physical health to facilitating social behavior (Mueller 2009; Mueller 2011; Gerling 2012). For this particular instance, these games allow for new and captivating social experiences either by inducing competitive edge or cooperation between players (Mueller 2009; Lindley 2008). Mueller has researched exergames extensively, exploring geographically distributed exertion activities and how users are able to motivate each other when the distance between them is a barrier for design (Mueller 2010).

Several other exergames exhibit different approaches towards persuading their users (Chittaro 2012). They rely on social pressure and on comparative data between the game's participants. Striving to become the best player or surpass a certain opponent is, in its essence, the same as accepting challenges from other individuals in commercially available exergames. Storytelling is an alternative way to entice players (Chittaro 2013), but one which falls outside the scope of our research.

\subsection{Social Presence}

Exercising with the aid of an exergame opens new scenarios and possibilities for individuals.
Traditionally, athletes were able to either engage in exercise alone or with a group of acquaintances. Exergames introduced a couple of more options into the landscape of possible participants during exercise. Among these, we emphasize the usage of virtual partners. We will now address each type of peer presence encountered in exergames.

\subsubsection{Physical}

Running alongside one or more individuals is a scenario which could take place before exergames were introduced. Reports show that people strive to find a regular running partner and even though there are specialized websites to find individuals to exercise with, people prefer doing it with their friends or acquaintances ${ }^{5}$. Thus, having a partner to which one can compare to can be pivotal towards steadily improving one's performance and motivation levels.

\subsubsection{Virtual}

Having a preferred exercising partner can have its share of benefits. However, it also introduces potential issues. Among these, the availability of both parties involved emerges as the most compromising. Videogames introduced the idea of using recordings of an individual's (or any of his / her friends or acquaintances) previous races. Typically denominated ghost recordings, these allow a user to keep awareness about whether his / her performance is better than that recording he / she is comparing to. Since then, this feature has been adopted in exergames extensively. This approach mitigates two problems raised by the need of physical presence: on the one hand, the mutual availability as far as the time of the exercise is concerned; on the other hand it allows for people who are geographically distributed to take part in comparison exercises via ghost recording sharing.

Fueled by the popularity of social networking services, exergames such as Nike+ or Endomondo broadened their focus to not only address the individuals actively exercising, but also those who may have interest in following / monitoring them in the comfort of their own homes / offices. During exercise activities, spectators can send cheering messages to people exercising. Usually, text-tospeech technology is employed to transform textual messages in audio cues. Alternatives to this approach include the usage of cheering / booing sounds to inform the user that he / she is being monitored remotely and that his / her performance is good / poor, respectively.

\footnotetext{
${ }^{5}$ Benefits of training partners:

http://running.competitor.com/2013/09/training/buddy-upthe-benefits-of-training-partners_58448
} 


\subsection{Conveying Live Performance Data}

Another aspect in which exergames converge is the way persuasive cues are delivered to users. Natural language is a common element in almost all of the studied exergames. The application typically issues an auditory message using natural language to motivate him / her. Music also has a strong presence in these applications. Personalized playlists appear to be a common denominator across exergames. Others extend this feature to include power-songs. Less popular approaches involve the usage of disruptions in a song to metaphorically signal loss of performance (Keep Running). The delivery of applause sounds is gaining momentum especially when spectators are involved.

\section{EXODUS}

In order to investigate how these three societal persuasive approaches impact on a user's perceived motivation and sense of competitiveness we envisioned and developed an exergame denominated ExodUS. We followed a user-centred design process, heavily rooted in the analysis of the most common functionalities shared between existing exergames. While the description of this process falls outside the scope of this contribution, we present the core feature-set which enabled the research hereby presented: how user effort is calculated and how the different types of presence are suported. ExodUS aims at hiking and jogging exercises, covering the same type of activities that Nike+ or Endomondo support and was developed for Android 2.1 (and above) devices. We must emphasize that ExodUS is an experimental prototype merely created to aid us exploring the role of physical and virtual presence, impeding it from being a major contribution of this research.

\subsection{Determining User Effort}

Measuring one's performance in an Olympic track is a straightforward task: the person with the best individual time wins. When the location chosen to practice is not a regulated one, the task is not as trivial. One may suggest using the distance covered: however, running uphill produces more strain than going downhill. A location independent metric is, thus, paramount to address this problem.

Tracking how much energy we expend during practice without specialized equipment is possible due to the variety of sensors integrated in modern smart-phones. Researchers have envisioned a set of formulas capable of giving this information with more or less accuracy. More accurate formulas rely on information such as the average heart rate during the exercise period. Within the context of ExodUS we opted for a simpler formula which takes into account the time, distance covered and altitude during the exercise activity according to the following criteria ${ }^{6}$ :

$$
\begin{array}{ll}
\text { - } & \text { Cal_DIS }=(\text { WEIGHT * } 0.75 * \text { DISTANCE }) \\
\text { - } & \text { Cal_INC }=(\text { WEIGHT * } 0.75 * \text { INCLINE }) \\
\text { - } & \text { TOTAL }=\text { Cal_DIS }+ \text { Cal_INC }
\end{array}
$$

Each formula takes into account a basic user characteristic (weight) and a performance feature (distance and incline, accordingly). With this, ExodUS is able to compare different users' performances and provide them with valuable information regarding their own progress (as utilized in the reported trials).

\subsection{Supporting Presence}

ExodUS encompasses functionalities to support different types of presence. We narrowed our focus towards virtual presence, comprising the ghosts and audience features.
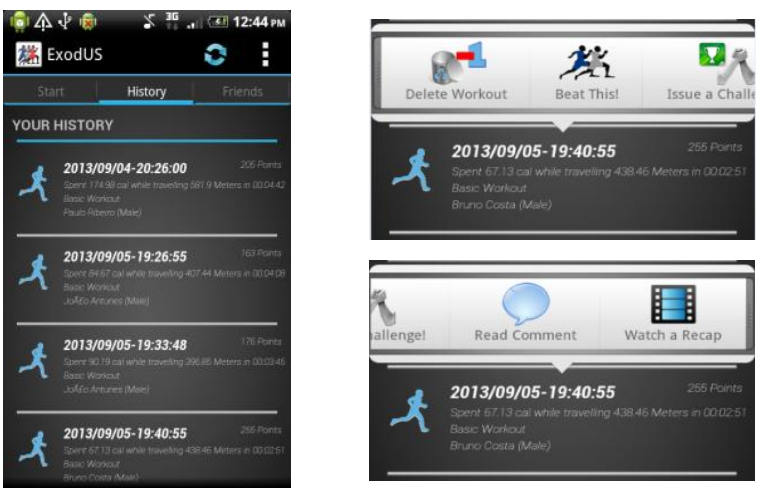

Figure 1 - ExodUS' ghost functionality interface.

\subsubsection{Virtual Ghost}

One of the social persuasion variants we employed in ExodUS addresses the usage of a virtual partner, henceforth referred to as a ghost. Ghosts are recordings of exercise activities which may or may not belong to the actual application user. In order for these to be properly used, both the location of the recording and the place where the user is currently training should be the same. To keep awareness regarding how the individual is faring against the ghost we opted to utilize audio feedback during the exercise activity (akin to what other exergames adopt). At a given moment (e.g. each 2 minutes, each $1000 \mathrm{~m}$ ) users are relayed feedback about their performance. This cadence is configurable by the user. We compare his / her energy expenditure and / or average speed with the ghost's and provide appropriate feedback for each situation (e.g. "You're gaining on him", "You're

6 Calories burned while jogging:

http://www.myfitnesspal.com/topics/show/134478accurate-formula-to-determine-calories-burned-jogging 
slower than your opponent", etc.), using a male voice from a text-to-speech synthesizer (Pico TTS).

Figure 1 depicts the ghost selection interface. On the left we can observe a list of ghost recordings which, upon selection, enable the player to challenge them, replay the ghost recording or comment, as seen on the right side.

\subsubsection{Virtual Audience}

The inclusion of virtual spectators contemplates two aspects which will be briefly explained. The first addresses the usage of appropriate cheering and booing sounds. These sounds were cautiously selected to inspire performing inside a crowded stadium. They are also conveyed to the user when: a) the user manually configures ExodUS to give him / her this type of feedback; b) when an authenticated user sends a cheering / booing message to the user. Users can also send text messages which are then translated via a TTS module to the user. ExodUS offers a social feature in which users may share their own performances with each other. We also implemented a monitoring feature which allows people in an individual's contact list to track exercising sessions live and send cheering / booing feedback to their friends. This is a web-based portal implemented using HTML5 and a MySQL database.

\section{EXPERIMENT}

We elaborated an experimental period to perform an early assessment of the impact of different types of presence on user perceived motivation and sense of competitiveness. The goal was to collect feedback from users that could be later used to fuel a proper design revamp of ExodUS to further explore distinct ways to persuade individuals.

\subsection{Methodology}

For this milestone of the research we opted to employ subjective assessment techniques using questionnaires to retrieve participants' perceptions.

\subsection{Participants}

15 participants (engineering course students; 12 male; 3 female; Age $M=27.7$; Age $S D=7.3$ ) volunteered to partake in these sessions. All participants exercised regularly and approximately $80 \%$ had had contact with exergames before.

\subsection{Tools \& Equipment}

Subjects were provided with an HTC Desire Android smart-phone loaded with the ExodUS application and a set of headphones to hear all feedback being conveyed. All users opted to carry it in their pockets. Lastly, they were offered with bottles of water to keep themselves fresh inbetween each trial.

\subsection{Procedure}

The experiment began with a profiling period to characterize each subject. Participants were provided with information concerning ExodUS (how it works, what type of data it infers, since subjects did not directly interact with it). The experiment then required each participant to carry out three tasks:

- Task 1 - perform a lap in an Olympic track with a real partner (RP). This partner changed pace throughout the race to induce feelings of uncertainty as of whether the subject was outperforming or underperforming. No subject was acquainted with this partner.

- Task 2 - perform a lap in an Olympic track using the ExodUS application pre-loaded with a virtual partner in the same track (VP). The ghost performed the track in 60 seconds. No real partner was used in this task. The ghost did not belong to the subject him / herself nor any acquaintance of him / her.

- Task 3 - perform a lap in an Olympic track using the ExodUS application with spectator mode on (virtual audience - VA). The user received cheering / booing cues as well as written messages conveyed via the audio channel periodically sent by a couple of persons who were not at the track. No real partner was present in this task. The subject had no knowledge regarding the audience identity.

Feedback received during Task 2 was issued based on the subjects' calorie expenditure as detailed in the previous section and was issued at fixed distance milestones (each 50 meters, totalling 8 feedback messages; content was based on user performance at the time). Task order was randomized for each individual, ensuring a balance between all combinations. There was a 10 minute interval between each task, during which participants were provided with refreshments offered by our team. At the end, we distributed a questionnaire which aimed to assess the subjects' perceptions on how each type of presence influenced their motivation and competitiveness levels. All answers were given scores using a Likert scale from 1 (lowest) to 5 (highest).

\subsection{Results}

The questionnaire's results can be observed in Figure 2 and Figure 3. 


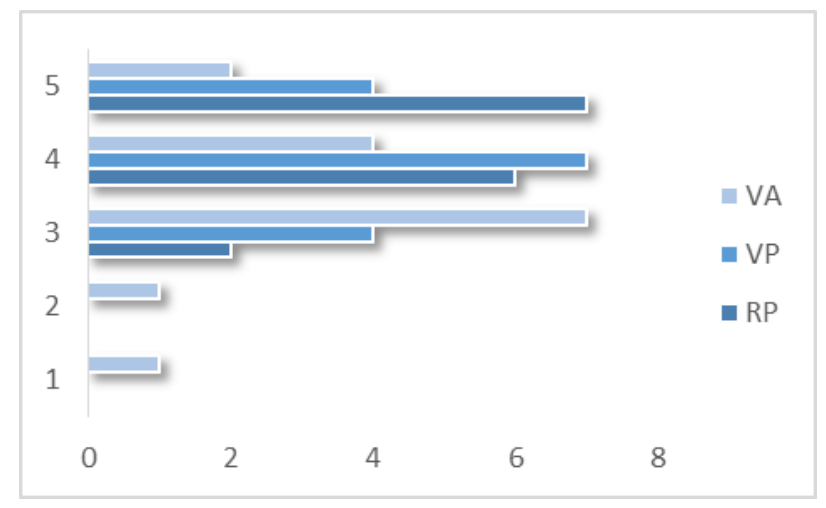

Figure 2 - Perceived motivation: XX axis - number of respondents; YY axis - Likert scale.

When asked "How much did the exercising with a real / virtual partner affect your motivation", users stated that the presence of the real partner (RP) had the most positive impact, with 7 participants stating it greatly motivated them. The usage of a virtual partner (VP), albeit slightly less positive, still manages to produce a noteworthy motivational impact on our subjects. Virtual audiences (VA) had the least positively impacting influence, with two users even stating they felt less motivated with the presence of that feature.

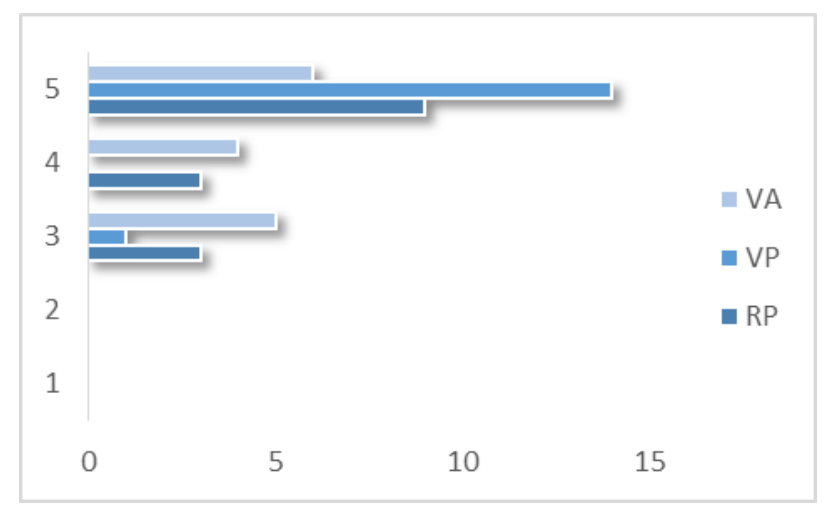

Figure 3 - Perceived Competitiveness: XX axis - number of respondents; YY axis - Likert scale..

When questioned about "How much did the exercising with a real / virtual partner affect your competitiveness", the most influencing presence types are switched. The virtual partner garners the choice of our subjects as the most impacting type of presence for their perceived competition level. Real partners also obtain a respectable approval rating. The usage of a virtual audience still fails to capture an overwhelmingly positive response from our population.

During the virtual audience trial, the audience members sent an average of 6 encouraging written messages (WM), 7 cheers and 5 boos to each participant $(\mathrm{C} / \mathrm{B})$. Figure 4 displays the perceived motivation impact results for the usage of written messages and cheering / booing sound cues. Written messages present an overall better appreciation concerning their motivational influence. While cheering / booing sounds do not perform poorly, opinions are mixed regarding its motivational value. These results are in line with Fogg's persuasion theories in which it is stated that the usage of natural language approaches can more easily reach users (Fogg 2002).

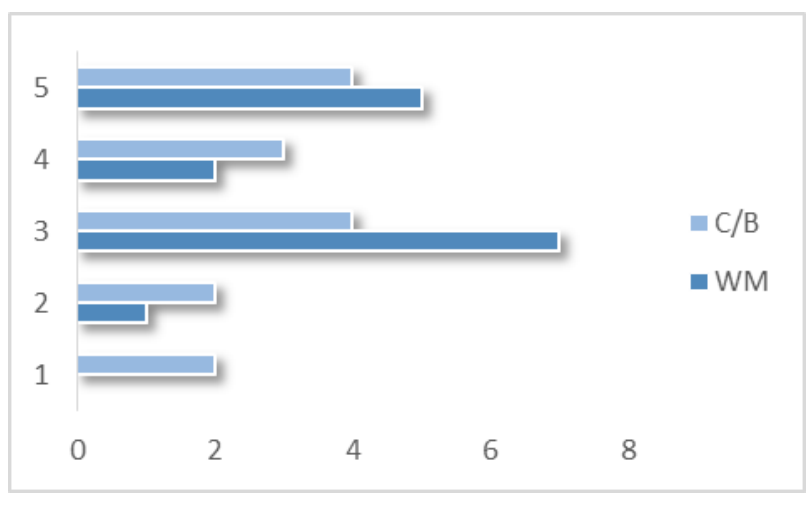

Figure 4-Perceived motivation impact from written messages and cheering / booing sound cues for virtual audiences: $X X$ axis - number of respondents; YY axis-Likert scale..

\section{DISCUSSION}

We will now address a few topics that are important to discuss not only within the context of this research, but also for possible future endeavours.

\subsection{Real VS Virtual}

Considering all of our research on this topic, the virtual ghost partner was expected to be the playing mode that created more impact on the players' motivation levels, since it is intrinsically linked with exercising alone and creating competition (most times against oneself). The results, although levelled, show that for this sample, exercising with a real world partner impacted players' motivation further. However, practicing alongside a virtual peer instilled a more emphasized competitive edge on athletes. Another interesting characteristic regarding the two is that running alongside a real partner made three participants feel more relaxed while that did not happen with the ghost challenge for any of the participants.

\subsection{Adjusting the Ghost}

Even though the impact of the ghost participant in both the users' motivation and perceived competitiveness is commendable, we believe there is room for improvement. The ghost used in the task stemmed from a performance run that one of the researchers had previously recorded. Inspired by the results reported by Mandryk (2004), we question whether players would feel more engaged if exercising against a friend's ghost rather than that of an unknown person. At the same time, we also dispute if different types of 
intonations or voice types could have an effect on the perceived motivation and competition levels.

\subsection{Creating an Enticing Audience}

Our virtual audience did not encompass any textto-speech technology. Instead, we opted to use cheering and booing sounds to evoke feelings of having a good or poor performance, respectively. A possible research direction involves the assessment of which types of sounds are most adequate to motivate users. Analysing the crowd size (number of people cheering / booing) can also be taken into account here.

\subsection{Study Limitations}

While the retrieved data is valuable on its own, not only to improve ExodUS' design, but also for other researchers and developers to learn more about how determined features affect a user's motivation and competitiveness levels, its scope is limited due to the preliminary nature of this stage of research.

The most important limitation is the design of the study itself. While we defend that giving almost no time to rationalize on their opinions is valuable to obtain honest answers, a longitudinal study would be more adequate. That option, however, also presents its limitations: it requires users who are willing to use the application regularly (or reward them to do so, increasing the cost of research). This leaves a slice of exergames potential user base out, since these applications are also developed aiming at users who do not exercise regularly and utilize the features we assessed in this article as a way to motivate them into doing so.

The number of participants is another caveat, aggravated by performing a single lap. Still, despite these caveats, we stand by this initial assessment paving the way for a more robust study in the future, given that the foundations for the next steps have hereby been validated.

\section{CONCLUSION \& FUTURE WORK}

In this preliminary assessment addressing the effects of different types of presence in mobile exertion applications we showed that virtual entities can have a similar influence as their real counterparts. Despite user sense of motivation being lower with virtual entities, the perceived competitiveness conveyed by virtual opponents was observed to be higher in the latter's case. Furthermore, we showed that virtual audiences, while less impactful, can still be a relevant feature in this type of applications. The type of feedback conveyed by those audiences is also capable of affecting users differently.
These findings were already incorporated into the latest ExodUS version. In the future we want to expand this research by conducting larger longitudinal studies to assess the long term effect of these presence types of user performance, motivation and social activity.

\section{ACKNOWLEDGEMENTS}

This work was partially supported by Fundação para a Ciência e Tecnologia (FCT) through multiannual funding to the LaSIGE research unit and Individual Scholarship SFRH/BD/39496/2007.

\section{REFERENCES}

Chittaro, L. et al (2012). Turning the classic Snake mobile game into a location-based exergame that encourages walking. In Proceedings of PERSUASIVE, 2012.

Chittaro, L. et al (2013). Exploring audio storytelling in mobile exergames to affect the perception of physical exercise. In Proceedings of Pervasive Health '13.

Duarte. L. et al (2014). Defining a Design Space for Persuasive Cooperative Interactions in Mobile Exertion Applications. In Proceedings of the 20th Conference on Collaboration and Technology, Santiago, Chile.

Fogg, BJ. (2002). Persuasive Technology: Using Computers to Change What We Think and Do. In Ubiquity Magazine.

Gerling, K. et al (2012). Full-body Motion-based Game Interaction for Older Adults. In Proceedings of the SIGCHI Conference on Human Factors in Computing Systems (CHI 2012). ACM, New York.

Lindley, Set al (2008). Stirring Up Experience Through Movement in Game Play: Effects on Engagement and Social Behavior. In Proceedings of the SIGCHI Conference on Human Factors in Computing Systems (CHI 2008). ACM, New York.

Mandryk, R. et al (2004). Physiological indicators for the evaluation of co-located collaborative play. In Proceedings of CSCW 2004.

Mueller, F., (2009). Exertion in Networked Games. In Proceedings of the 4th International Conference on Foundation of Digital Games.

Mueller, F. et al (2010). Jogging over a distance between Europe and Australia. In Proceedings of UIST '10. ACM, 189-198.

Mueller, F. et al (2011). Designing Sports: A Framework for exertion Games. In Proceedings of CHI 2011. 